\title{
From entanglement certification with quench dynamics to multipartite entanglement of interacting fermions
}

\author{
Ricardo Costa de Almeida $[$ and Philipp Hauke $\mathbb{0}$ \\ INO-CNR BEC Center and Department of Physics, University of Trento, Via Sommarive 14, I-38123 Trento, Italy; \\ Kirchhoff Institute for Physics, Ruprecht Karl University of Heidelberg, Im Neuenheimer Feld 227, D-69120 Heidelberg, Germany; \\ and Institute for Theoretical Physics, Ruprecht Karl University of Heidelberg, Philosophenweg 16, D-69120 Heidelberg, Germany
}

(Received 3 June 2020; revised 26 January 2021; accepted 1 June 2021; published 24 August 2021)

\begin{abstract}
Multipartite entanglement, such as witnessed through the quantum Fisher information (QFI), is a crucial resource for quantum technologies, but its experimental certification is highly challenging. Here, we propose an experimentally friendly protocol to measure the QFI. It relies on recording the short-time dynamics of simple observables after a quench from a thermal state, works for spins, bosons, and fermions, and can be implemented in standard cold-atom experiments and other platforms with temporal control over the system Hamiltonian. To showcase the protocol, we simulate it for the one-dimensional Fermi-Hubbard model. Further, we establish a family of bounds connecting the QFI to multipartite mode entanglement for fermionic systems, which enable the detection of multipartite entanglement at sizable temperatures. Our work paves a way to experimentally accessing entanglement for quantum enhanced metrology.
\end{abstract}

DOI: 10.1103/PhysRevResearch.3.L032051

\section{INTRODUCTION}

A central question for quantum many-body physics is to understand the structure of entanglement and how it translates into observable features. Besides its potential to explain certain salient many-body phenomena [1-6], it may take a decisive role as a resource in upcoming quantum technologies. Hence, as these technologies mature, scalable protocols for detecting entanglement become increasingly necessary $[7,8]$. This demand is already a reality for quantum metrology [9] where the quantum Fisher information (QFI) [10], a witness for multipartite entanglement, determines the metrological quantum enhancement [11-14]. Although lower bounds of the QFI have been obtained in recent groundbreaking experiments [15-18], general and efficient procedures to directly extract its precise value in many-body systems are lacking.

To tackle this challenge, we develop an experimentally accessible technique for measuring the QFI for states in thermal equilibrium. In contrast to a previous proposal relying on frequency-dependent dynamic susceptibilities [19], our protocol only requires measuring the short-time dynamics of mean expectation values after a quench. This straightforward procedure is highly error resilient and is ideally suited, e.g., for standard experiments on ultracold atoms [20-24] but can also be applied in other platforms. This measurement protocol for the QFI is our first main result.

Moreover, previous studies about the QFI and entanglement bounds have focused on systems describable as spins

Published by the American Physical Society under the terms of the Creative Commons Attribution 4.0 International license. Further distribution of this work must maintain attribution to the author(s) and the published article's title, journal citation, and DOI.
[11-13]. Nonetheless, interacting fermions are of central importance to condensed matter physics and experiments with ultracold atoms have enabled the precise engineering of fermionic many-body systems [25-31].

Motivated by this, we derive bounds that relate multipartite fermionic mode entanglement to the QFI, by generalizing the concept of $k$ producibility to fermionic systems. This framework for fermionic multipartite entanglement is our second main result. It complements the existing literature that is focused on spin models, can be applied to any system described with the second quantization formalism, and proves that mode entanglement provides quantum enhancement of metrological devices.

We illustrate these bounds as well as our quench-based measurement protocol for the QFI at a paradigmatic example, the Fermi-Hubbard model in one dimension (1D). As shown in Fig. 1 and discussed further below, we certify the presence of multipartite mode entanglement for a broad region of the parameter space.

The article is organized as follows: First, we review some basic notions regarding the QFI. We proceed to derive our quench protocol. Afterwards, we rigorously define multipartite mode entanglement for fermions and determine the correct fermionic entanglement bounds for the QFI. Subsequently, we discuss the results shown in Fig. 1 in detail and provide a guideline to experiments aiming at certifying entanglement in the Fermi-Hubbard model. We conclude the article with a brief outlook.

\section{BACKGROUND ON THE QFI}

The quantum Fisher information, $F_{\mathrm{Q}}[\rho, O]$, is a central concept in quantum metrology. It quantifies the metrological sensitivity obtained from a given quantum state $\rho$ in a phase estimation setup, in which a unitary generated by an 


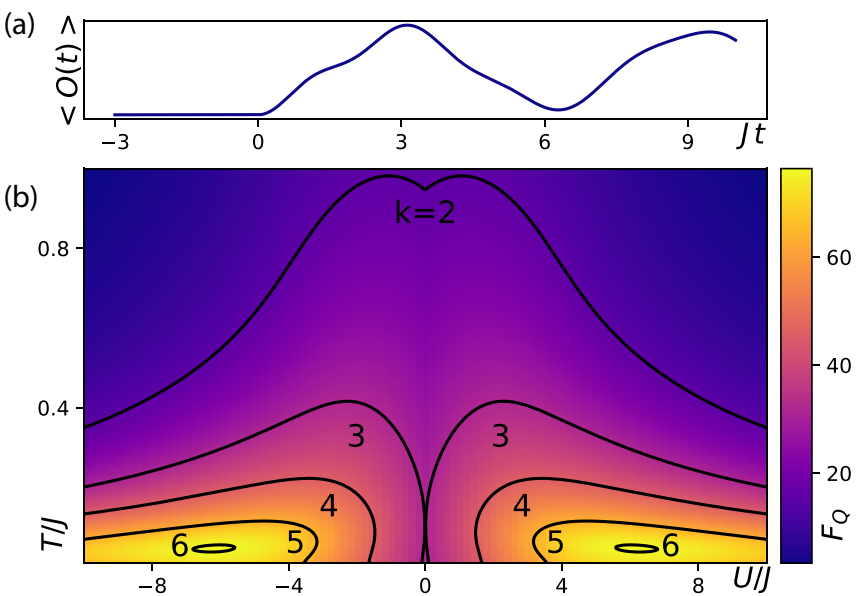

FIG. 1. Certification of multipartite mode entanglement. (a) A many-body quantum system is abruptly perturbed by an operator $O$. Subsequently, the evolution of the same observable $\langle O(t)\rangle$ is measured, from which the quantum Fisher information, $F_{\mathrm{Q}}[\rho, O]$, is extracted. (b) $F_{\mathrm{Q}}[\rho, O]$, computed by simulating the protocol for the Fermi-Hubbard model in 1D. Using the entanglement bounds of Eq. (1) (contour lines), many-body entanglement is certified up to large temperatures. Data for $L=8$ and open boundary conditions.

operator $O$ rotates $\rho$ by an angle $\theta, \rho(\theta)=e^{-i \theta O} \rho e^{i \theta O}$ [32]. The aim in this scenario is to precisely estimate the parameter $\theta$, whose variance after $m$ measurements is bounded through the Crameér-Rao bound, $\operatorname{Var} \theta \geqslant 1 /\left(m F_{\mathrm{Q}}[\rho, O]\right)[10]$.

Moreover, the QFI witnesses multipartite entanglement. Specifically, we show this below for fermionic states $\rho$ characterized by fermionic modes labeled by a set $M$. If $\rho$ satisfies

$$
F_{\mathrm{Q}}[\rho, O]>\left(d k^{2}+r^{2}\right),
$$

it must be, at least, $(k+1)$-partite mode entangled where $d=\lfloor M / k\rfloor$ denotes the integer division of $|M|$ by $k$ and $r=$ $|M|-d k$ is the remainder of the division. This result complements existing, analogous bounds for spin systems [11-13]. Intuitively, the higher correlations of an entangled many-body state, relative to a classical state, lead to a greater sensitivity to perturbations and thus to greater metrological gain, quantified through $F_{\mathrm{Q}}[\rho, O]$. In particular, in a separable state $F_{\mathrm{Q}}[\rho, O]$ is bounded by $|M|$, the scaling observed in classical systems, whereas any metrological enhancement beyond the classical limit requires entanglement. This enables the use of $F_{\mathrm{Q}}[\rho, O]$ for entanglement certification.

The QFI of a pure state $\rho=|\psi\rangle\langle\psi|$ is simply a variance, $F_{\mathrm{Q}}[\rho, O]=4 \operatorname{Var} O=4\left(\left\langle\psi\left|O^{2}\right| \psi\right\rangle-\langle\psi|O| \psi\rangle^{2}\right)$, so it can be calculated efficiently. However, the formula for an arbitrary density matrix $\rho=\sum_{\lambda} \rho_{\lambda}|\lambda\rangle\langle\lambda|$,

$$
F_{\mathrm{Q}}[\rho, O]=2 \sum_{\lambda, \lambda^{\prime}} \frac{\rho_{\lambda}-\rho_{\lambda^{\prime}}}{\rho_{\lambda}+\rho_{\lambda^{\prime}}}\left(\rho_{\lambda}-\rho_{\lambda^{\prime}}\right)\left|\left\langle\lambda|O| \lambda^{\prime}\right\rangle\right|^{2},
$$

requires diagonalizing the state, which is a challenging undertaking for quantum many-body systems, both theoretically and experimentally. In what follows, we show how to circumvent this difficulty by extracting the QFI for thermal states from expectation values using a quench.

\section{DERIVATION OF THE QUENCH PROTOCOL}

In a previous work [19], a connection between the QFI and linear response theory was found that enables one to compute the QFI for systems in equilibrium at temperature $T$,

$$
F_{\mathrm{Q}}[\rho, O]=\frac{4}{\pi} \int_{0}^{+\infty} d \omega \tanh \left(\frac{\omega}{2 T}\right) \chi^{\prime \prime}(\omega, T) .
$$

This formula requires knowledge of $\chi^{\prime \prime}(\omega, T)=\mathfrak{s}(\chi(\omega, T))$, the imaginary part of the Fourier transform of the response function

$$
\chi(t-\tau, T)=\theta(t-\tau)\langle[O(t), O(\tau)]\rangle .
$$

This function characterizes the linear response of an observable $\langle O(t)\rangle$ to a time-dependent perturbation from $H_{0}$ to $H(t)=H_{0}-f(t) O$, where $H_{0}$ is the Hamiltonian with respect to which the system was at thermal equilibrium. For deviations $\Delta O(t)$ from the equilibrium value, the Kubo formula gives [33]

$$
\begin{aligned}
\Delta O(t) & =\langle O(t)\rangle-\langle O\rangle=(\chi * f)(t) \\
& =\int_{-\infty}^{+\infty} \mathrm{d} \tau \chi(t-\tau, T) f(\tau) .
\end{aligned}
$$

By transforming the integral from frequency to time domain, an equation analogous to Eq. (3) follows as

$$
F_{\mathrm{Q}}[\rho, O]=4 T \int_{0}^{+\infty} \mathrm{d} t \frac{\chi(t, T)}{\sinh (\pi t T)},
$$

which allows the QFI to be obtained directly from the Kubo response function. The time domain expression has computational advantages compared to Eq. (3) and has been used for computing the QFI [19,34].

Conceptually, these expressions represent a significant advance as they explicitly relate the QFI to correlations encoded in the response functions. However, their application still presents practical problems as measurements of unequal-time correlation functions, such as $\langle[O(t), O]\rangle$, are often challenging. Very recently, the protocol based on Eq. (3) has revealed the scaling of the QFI using neutron scattering [35], but detection of the absolute level of multipartite entanglement in this way has not been achieved yet.

We overcome such limitations by introducing a protocol that solely relies on measurements of expectation values $\langle O(t)\rangle$. To realize such a simplified protocol only requires a weak, abrupt quench, as can be conveniently implemented, e.g., in cold-atom experiments [21-24]. In this scenario, the drive function is simply $f(\tau)=q \theta(\tau)$, so, from Eq. (5), the dynamics are governed by

$$
\Delta O(t)_{\text {quench }}=q \int_{0}^{t} \mathrm{~d} \tau \chi(\tau, T)=q \xi(t, T) .
$$

Here, $q$ denotes the quench amplitude and we introduced $\xi(t, T)=\Delta O(t)_{\text {quench }} / q$. Using $\chi(t, T)=\mathrm{d} \xi(t, T) / \mathrm{d} t$ in Eq. (6), we arrive at

$$
F_{\mathrm{Q}}[\rho, O]=\frac{4 \pi T^{2}}{q} \int_{0}^{+\infty} \mathrm{d} t \frac{\Delta O(t)_{\mathrm{quench}}}{\sinh (\pi t T) \tanh (\pi t T)}
$$




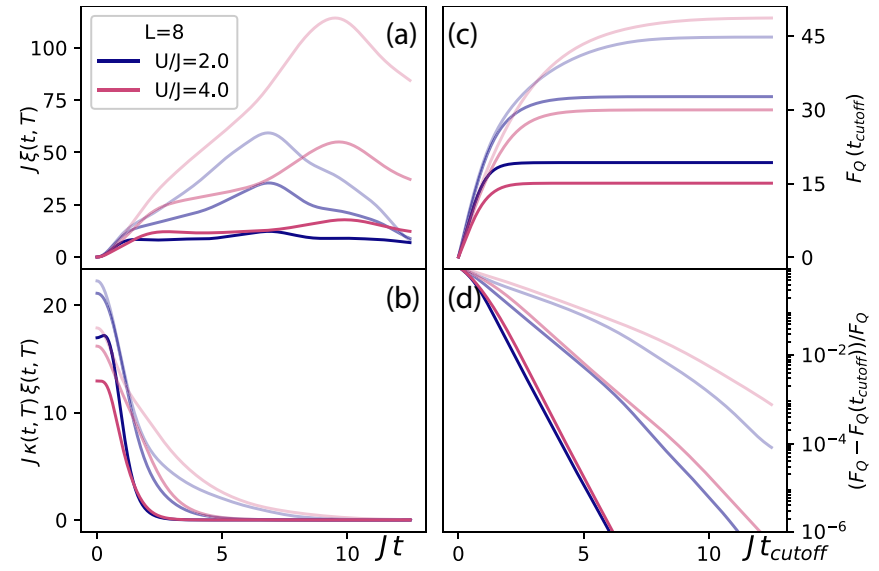

FIG. 2. Quench protocol for QFI extraction, exemplified for the Fermi-Hubbard model at temperatures $T / J=0.2,0.4,0.8$ (from light to dark shades) for a quench with the staggered magnetization [Eq. (16)]. (a) At time $t=0$, the system is quenched with the operator $O$ and strength $q$. Measuring the deviations from the equilibrium expectation value yields $\xi(t, T)=\Delta O(t)_{\text {quench }} / q$. (b) Using Eq. (8), the QFI can be computed by integrating $\xi(t, T)$ multiplied with the kernel function, $\kappa(t, T)$. (c) Cutting the integral off at time $t_{\text {cutoff }}$ produces a lower bound $F_{\mathrm{Q}}[\rho, O]\left(t_{\text {cutoff }}\right) \leqslant F_{\mathrm{Q}}[\rho, O]$. (d) Due to the functional form of $\kappa(t, T)$, the convergence is exponentially fast with a decay constant set by the temperature, $F_{\mathrm{Q}}[\rho, O]-$ $F_{\mathrm{Q}}[\rho, O]\left(t_{\text {cutoff }}\right) \sim \exp \left(-\pi T t_{\text {cutoff }}\right)$.

after performing an integration by parts and handling the convergence issues that arise. See the Supplemental Material [36] for details.

From Eq. (8), we can summarize our protocol by four steps (see also Fig. 2): (i) Prepare a thermal state. (ii) Turn on quench. (iii) Measure dynamics of expectation values. (iv) Integrate results according to Eq. (8).

Thermal equilibrium and a quench in the linear regime are the only assumptions used for deriving Eq. (8), so the protocol applies to arbitrary quench operators and quantum many-body systems, including fermionic, bosonic, and spin systems. Critically, only the expectation value of $O(t)$ is used and access to higher moments is not necessary. Moreover, it has a series of further advantageous properties. For example, it simplifies the requirements for extracting the QFI in many situations, as no time-time correlations are required, and the exponential decrease of $\kappa(t, T)=4 \pi T^{2}[\sinh (\pi t T) \tanh (\pi t T)]^{-1}$ with time implies only short measurement times are required. This feature provides resilience against dissipative effects such as atom loss, see Supplemental Material [36]. At small temperatures, where the required observation times become long, the variance of $O$ in the initial state yields a reliable upper bound on the QFI. Our protocol can complement this by a lower bound (see below).

Further, the requirement of quenching in the linear regime can be tested by comparing responses with different $q$ parameters, which also enables extrapolation to $q=0$. In the Supplemental Material [36], we discuss nonlinear effects using our exact numerics. For the considered model, values as large as $q=0.1 \mathrm{~J}$ produce only deviations of a few percent and, critically, do not generate any false positives.

\section{ENTANGLEMENT BOUNDS FOR FERMIONIC SYSTEMS}

Based on the concept of $k$ producibility, for spin systems bounds on the QFI have been derived that only states with multipartite entanglement can overcome [11-13]. However, for fermionic systems, such bounds do not exist.

To remedy this situation, we first need to adapt the notion of $k$ producibility. To see why this is necessary, we recall the condition for a state of $N$ spins to be $k$ producible,

$$
|\psi\rangle_{k \text {-prod. }}^{\text {spin }}=\left|\psi_{1}\right\rangle \otimes\left|\psi_{2}\right\rangle \otimes \cdots \otimes\left|\psi_{P-1}\right\rangle \otimes\left|\psi_{P}\right\rangle,
$$

where $\left|\psi_{j}\right\rangle$ is a state of $N_{j} \leqslant k$ spins and $\sum_{j} N_{j}=N$. Such a decomposition is not meaningful in the fermionic case due to the antisymmetric structure of the wave function. Fortunately, this also suggests what is the correct criteria, which we now introduce.

Consider a set of fermionic modes $M$, with associated creation and annihilation operators $c_{m}^{\dagger}$ and $c_{m}$, labeled by $m \in M$. A $k$ partition of the system is defined as a partition $M=M_{1} \cup M_{2} \cup \cdots \cup M_{P}$ subject to $\left|M_{j}\right| \leqslant k$. Now, we introduce the following definition: a pure fermionic state $|\psi\rangle$ is $k$ producible if there is a $k$ partition such that

$$
|\psi\rangle_{k \text {-prod. }}=C_{1}^{\star} C_{2}^{\star} \ldots C_{P}^{\star}|\rangle,
$$

where the operator $C_{j}^{\star}$ is restricted to act on $M_{j}$. The $C_{j}^{\star}$ can be written as linear combinations of products of creation operators $c_{m}^{\dagger}$ acting within $M_{j}$,

$$
C_{j}^{\star}=\sum_{\eta_{j}} \phi_{j}^{\star}\left(\eta_{j}\right) \prod_{m \in M_{j}}\left(c_{m}^{\dagger}\right)^{\eta_{j}(m)} .
$$

Here, without loss of generality, we fix some order for applying the creation operators. The summands are labeled by numbers $\eta_{j}(m) \in\{0,1\}$, which one can envision as the possible occupations of the modes, with associated amplitudes $\phi_{j}^{\star}\left(\eta_{j}\right) \in \mathbb{C}$.

An explicit connection with the spin definition is possible if we introduce $\left|\psi_{j}\right\rangle=C_{j}^{\star}|\rangle$ and notice that Eq. (10) can be written as $|\psi\rangle \sim\left|\psi_{1}\right\rangle \wedge\left|\psi_{2}\right\rangle \wedge \cdots \wedge\left|\psi_{P}\right\rangle$, with the exterior product $\wedge$ acting as an antisymmetric analog of the tensor product. The 1-producible decomposition with the exterior product has been used before to study mode entanglement in fermionic systems [37]. Nonetheless, for our purposes the operator language as in Eq. (11) is more convenient. The same formulation can be adapted to bosonic and spin systems, where it reproduces the usual definition of $k$-producible states. For 2-partite entanglement, our definition is equivalent to the one through the Slater number [38,39].

The extension of these concepts to mixed states $\rho$ is standard [11-13]: a mixed state $\rho_{k \text {-sep. }}$ is $k$ separable if it can be written as a convex hull

$$
\rho_{k \text {-sep. }}=\sum_{\lambda} \rho_{\lambda}|\lambda\rangle_{k \text {-prod. }}\left\langle\left.\lambda\right|_{k \text {-prod. }}\right.
$$

of $k$-producible states $|\lambda\rangle_{k \text {-prod. }}$ This formulation introduces a hierarchy for mixed states that defines multipartite entanglement of fermionic modes: a state is $(k+1)$-partite mode entangled if it is not $k$ separable.

Using this notion, we can now establish bounds on multipartite mode entanglement. To connect to the QFI, we focus 
on operators of the form

$$
O=\sum_{m \in M} w(m) c_{m}^{\dagger} c_{m}
$$

with $w(m) \in \mathbb{R}$ weighting the occupation of different modes. Given a $k$-producible state $\rho=|\psi\rangle_{k \text {-prod. }}\left\langle\left.\psi\right|_{k \text {-prod. }}\right.$, one can define probability distributions $p_{j}\left(\eta_{j}\right)=\left|\phi_{j}\left(\eta_{j}\right)\right|^{2}$ for the $\eta_{j}$ and associated random variables $w_{j}\left(\eta_{j}\right)=$ $\sum_{m \in M_{j}} w(m) \eta_{j}(m)$ such that $F_{\mathrm{Q}}[\rho, O]=4 \sum_{j} \operatorname{Var} w_{j}$. Employing Popoviciu's inequality [40] to bound $\operatorname{Var} w_{j}$, it follows that

$$
F_{\mathrm{Q}}[\rho, O] \leqslant 4 \sum_{j} \frac{1}{4}\left(\max _{\eta_{j}} w_{j}\left(\eta_{j}\right)-\min _{\eta_{j}} w_{j}\left(\eta_{j}\right)\right)^{2} .
$$

Additional knowledge about the state $|\psi\rangle_{k \text {-prod. }}$ leads to restrictions on the allowed occupations $\eta_{j}$ and permits the derivation of tighter bounds for Eq. (14). In particular, if $|\psi\rangle_{k \text {-prod. }}$ has a fixed occupation number

$$
F_{\mathrm{Q}}[\rho, O] \leqslant \frac{d k^{2}+r^{2}}{4}\left(\max _{m} w(m)-\min _{m} w(m)\right)^{2},
$$

where we used the decomposition $|M|=d k+r$. See the Supplemental Material [36] for a detailed discussion and tighter bounds for the case where the occupation number is known.

Equation (15) and related bounds immediately extend to $k$-separable mixed states due to the convexity of $F_{\mathrm{Q}}[\rho, O]$. As a consequence, any state that overcomes this bound cannot be $k$ separable and must be, at least, $(k+1)$-partite mode entangled.

Different choices of the modes $M$ lead to different bounds. For the results presented here, we choose to work on the realspace site mode basis, since then the entanglement bounds are directly connected to the concept of locality. Nevertheless, our methods is also applicable to other bases, e.g., modes of the reciprocal space [41].

\section{RESULTS FOR FERMI-HUBBARD CHAIN}

We illustrate our main results on the 1D Fermi-Hubbard model, a paradigmatic model for an interacting, fermionic many-body system. Its Hamiltonian reads

$$
H_{0}=-J \sum_{x, \sigma}\left(c_{\sigma x}^{\dagger} c_{\sigma x+1}+\text { h.c. }\right)+U \sum_{x}\left(c_{\downarrow x}^{\dagger} c_{\downarrow x} c_{\uparrow x}^{\dagger} c_{\uparrow x}\right) .
$$

The fermions live on lattice sites $x=1,2, \ldots L$ and have two internal states, $\sigma=\uparrow, \downarrow$. $J$ governs hopping between neighboring sites and $U$ controls on-site interactions. The Hamiltonian $H_{0}$ commutes with total occupation and magnetization, and we choose to work on the magnetization-free subspace at half-filling.

To evaluate the QFI via Eq. (8), we consider quenches using the staggered magnetization, $O_{+}$, and density, $O_{-}$,

$$
O_{ \pm}=\sum_{x}(-1)^{x}\left(c_{\uparrow x}^{\dagger} c_{\uparrow x} \mp c_{\downarrow x}^{\dagger} c_{\downarrow x}\right)
$$

This choice is motivated by limit cases: at $U \rightarrow+\infty$ and half-filling, the fermions form a Néel state with homogeneous density and alternating internal state. Here, $O_{+}$differentiates

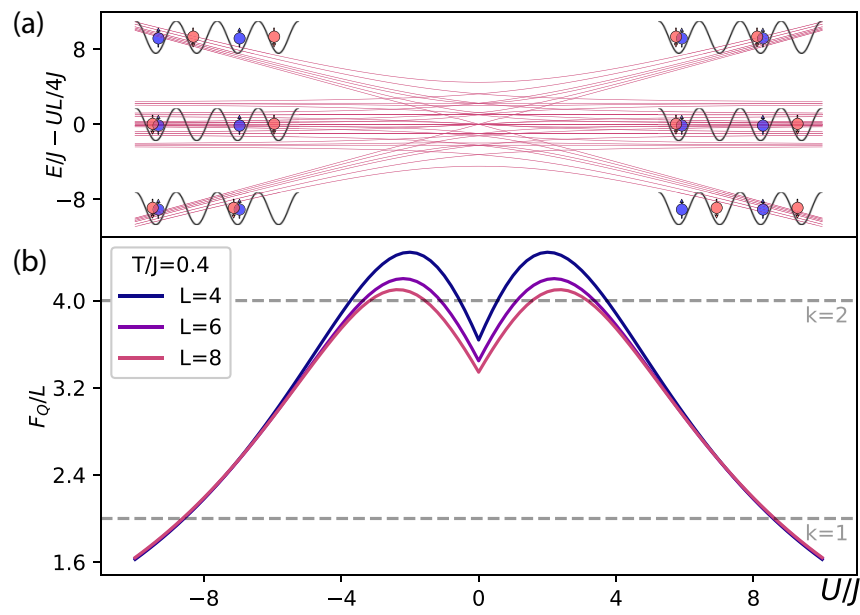

FIG. 3. Signature of robust entanglement in a Fermi-Hubbard $1 \mathrm{D}$ chain. (a) Spectrum of the model, exemplified for $L=4$. At $U / J \rightarrow 0$, the system is a free theory with regular level spacings. The bands for $U / J \rightarrow-\infty(U / J \rightarrow \infty)$ are a direct signature of the effective antiferromagnetic description. Higher bands correspond to breaking of pairs (creation of doublon-holon excitations). This picture falls apart for intermediate $U$ as the the system moves out of the perturbative regime and displays strongly correlated behavior. (b) $F_{\mathrm{Q}}[\rho, O]$ density for different system sizes with thresholds for certifying entanglement(dotted lines). The breakdown of the effective theory coincides with an increase in the robustness of the entanglement certified against thermal effects.

between the two degenerate ground states describing two possible alternating orders. For $U \rightarrow-\infty$, the fermions pair up to form a charge-density wave with homogeneous magnetization. Here, $O_{-}$distinguishes two possibilities of alternating large and low density. These limiting situations can be described analytically by an effective antiferromagnetic theory [31]. Based on intuition about which operators are expected to show strong quantum fluctuations [19], we expect $O_{ \pm}$to give a large QFI as one goes from the free theory at $U / J \rightarrow 0$ to the antiferromagnetic limit. In the Supplemental Material [36], we numerically confirm that $O_{ \pm}$are optimal for the considered scenario.

To simulate the quench protocol, we extract $\xi(t, T)$ from exact diagonalization and use it to calculate $F_{\mathrm{Q}}[\rho, O]$, taking the larger one of $F_{\mathrm{Q}}\left[\rho, O_{+}\right]$and $F_{\mathrm{Q}}\left[\rho, O_{-}\right]$. The results are summarized in Figs. 1 and $3 . F_{\mathrm{Q}}[\rho, O]$ increases rapidly as one moves away from the noninteracting point. In particular, in the intermediate region, where neither the free nor the antiferromagnetic theory describes the system, multipartite entanglement is detected at temperatures as large as $T / J=$ 0.4 . The system-size dependence suggests the entanglement to be especially robust in this strongly interacting region, making it a prime candidate to search for experimental signatures of multipartite entanglement.

Figure 4 illustrates how one can straightforwardly realize the quenches with $\mathrm{O}_{-}$and $\mathrm{O}_{+}$using optical superlattices. Ultracold atoms are now reaching strongly correlated manybody states of the Fermi-Hubbard model at temperatures as low as $T / J=0.25$ [43-45], well within the region where multipartite entanglement can be detected (see Fig. 1). Moreover, 


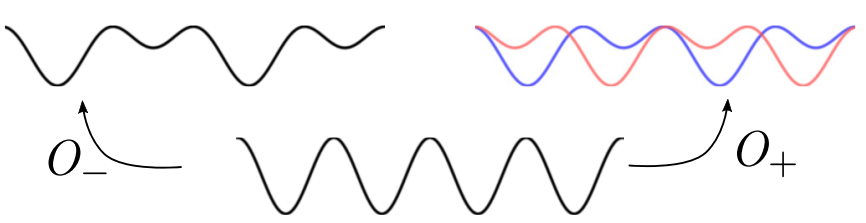

FIG. 4. Lattice quench in the Fermi-Hubbard model. A quench with $O_{ \pm}$amounts to abruptly modifying the chemical potential in a staggered fashion, which can be simply implemented through superlattices, which are spin-dependent for $O_{+}$, without the need for quantum gas microscopes. The relevant observable $\left\langle O_{ \pm}(t)\right\rangle$ can be measured through site-dependent imaging [42].

as shown in Figs. 2(c) and 2(d), at such temperatures the QFI converges within few hopping events $(J t \lesssim 8)$, i.e., on time scales faster than typical decoherence rates [45]. Thus, our quench protocol enables the detection of multipartite entanglement within existing experimental setups.

\section{CONCLUSION}

Though discussed in the context of ultracold fermionic gases, the simplicity and generality of our protocol make it readily applicable across different platforms. For instance, in solid-state systems off-diagonal elements of the QFI matrix can be extracted in quantum Hall samples (see Supplemental Material [36]). It is also straightforward to treat more intricate models, for instance, by adding further modes that describe higher bands, and to replace the simple quench we have chosen by other time-dependent functions $f(t)$, which just requires modifying the kernel function $\kappa(t, T)$ (see Supplemental Material [36] ). Recent works have studied the dynamical behavior of the quantum Fisher information after a quantum quench $[46,47]$. Here, turning things on their head, we have demonstrated the power of induced dynamics to extract the quantum Fisher information. Beyond the setup developed here, there is the possibility of applying our protocol to different thermodynamical ensembles [48] and even extend it outside the realm of thermodynamical states [49].

\section{ACKNOWLEDGMENTS}

This work is part of and supported by the DFG Collaborative Research Centre "SFB 1225 (ISOQUANT)", the Provincia Autonoma di Trento, and the ERC Starting Grant StrEnQTh (Project-ID 804305). Q@TN, the joint lab between University of Trento, FBK-Fondazione Bruno Kessler, INFNNational Institute for Nuclear Physics, and CNR-National Research Council.
[1] L. Amico, R. Fazio, A. Osterloh, and V. Vedral, Entanglement in many-body systems, Rev. Mod. Phys. 80, 517 (2008).

[2] N. Laflorencie, Quantum entanglement in condensed matter systems, Phys. Rep. 646, 1 (2016).

[3] G. D. Chiara and A. Sanpera, Genuine quantum correlations in quantum many-body systems: A review of recent progress, Rep. Prog. Phys. 81, 074002 (2018).

[4] D. A. Abanin, E. Altman, I. Bloch, and M. Serbyn, Colloquium: Many-body localization, thermalization, and entanglement, Rev. Mod. Phys. 91, 021001 (2019).

[5] C. Gogolin and J. Eisert, Equilibration, thermalisation, and the emergence of statistical mechanics in closed quantum systems, Rep. Prog. Phys. 79, 056001 (2016).

[6] X.-G. Wen, Colloquium: Zoo of quantum-topological phases of matter, Rev. Mod. Phys. 89, 041004 (2017).

[7] O. Gühne and G. Tóth, Entanglement detection, Phys. Rep. 474, 1 (2009).

[8] N. Friis, G. Vitagliano, M. Malik, and M. Huber, Entanglement certification from theory to experiment, Nat. Rev. Phys. 1, 72 (2018).

[9] L. Pezzè, A. Smerzi, M. K. Oberthaler, R. Schmied, and P. Treutlein, Quantum metrology with nonclassical states of atomic ensembles, Rev. Mod. Phys. 90, 035005 (2018).

[10] S. L. Braunstein and C. M. Caves, Statistical Distance and the Geometry of Quantum States, Phys. Rev. Lett. 72, 3439 (1994).

[11] L. Pezzé and A. Smerzi, Entanglement, Nonlinear Dynamics, and the Heisenberg Limit, Phys. Rev. Lett. 102, 100401 (2009).

[12] G. Tóth, Multipartite entanglement and high-precision metrology, Phys. Rev. A 85, 022322 (2012).

[13] P. Hyllus, W. Laskowski, R. Krischek, C. Schwemmer, W. Wieczorek, H. Weinfurter, L. Pezzé, and A. Smerzi, Fisher information and multiparticle entanglement, Phys. Rev. A 85 , 022321 (2012).

[14] G. Tóth and I. Apellaniz, Quantum metrology from a quantum information science perspective, J. Phys. A 47, 424006 (2014).

[15] H. Strobel, W. Muessel, D. Linnemann, T. Zibold, D. B. Hume, L. Pezze, A. Smerzi, and M. K. Oberthaler, Fisher information and entanglement of non-Gaussian spin states, Science 345, 424 (2014).

[16] B. Lucke, M. Scherer, J. Kruse, L. Pezze, F. Deuretzbacher, P. Hyllus, O. Topic, J. Peise, W. Ertmer, J. Arlt, L. Santos, A. Smerzi, and C. Klempt, Twin matter waves for interferometry beyond the classical limit, Science 334, 773 (2011).

[17] B. Lücke, J. Peise, G. Vitagliano, J. Arlt, L. Santos, G. Tóth, and C. Klempt, Detecting Multiparticle Entanglement of Dicke States, Phys. Rev. Lett. 112, 10.1103155304 (2014).

[18] J. G. Bohnet, B. C. Sawyer, J. W. Britton, M. L. Wall, A. M. Rey, M. Foss-Feig, and J. J. Bollinger, Quantum spin dynamics and entanglement generation with hundreds of trapped ions, Science 352, 1297 (2016).

[19] P. Hauke, M. Heyl, L. Tagliacozzo, and P. Zoller, Measuring multipartite entanglement through dynamic susceptibilities, Nat. Phys. 12, 778 (2016).

[20] I. Bloch, Ultracold quantum gases in optical lattices, Nat. Phys. 1, 23 (2005).

[21] M. Lewenstein, A. Sanpera, V. Ahufinger, B. Damski, A. Sen(De), and U. Sen, Ultracold atomic gases in optical lattices: mimicking condensed matter physics and beyond, Adv. Phys. 56, 243 (2007).

[22] P. Hauke, F. M. Cucchietti, L. Tagliacozzo, I. Deutsch, and M. Lewenstein, Can one trust quantum simulators?, Rep. Prog. Phys. 75, 082401 (2012). 
[23] J. Eisert, M. Friesdorf, and C. Gogolin, Quantum many-body systems out of equilibrium, Nat. Phys. 11, 124 (2015).

[24] T. Langen, R. Geiger, and J. Schmiedmayer, Ultracold atoms out of equilibrium, Annu. Rev. Condens. Matter Phys. 6, 201 (2015).

[25] S. Giorgini, L. P. Pitaevskii, and S. Stringari, Theory of ultracold atomic fermi gases, Rev. Mod. Phys. 80, 1215 (2008).

[26] T. Esslinger, Fermi-hubbard physics with atoms in an optical lattice, Annu. Rev. Condens. Matter Phys. 1, 129 (2010).

[27] F. Serwane, G. Zurn, T. Lompe, T. B. Ottenstein, A. N. Wenz, and S. Jochim, Deterministic preparation of a tunable fewfermion system, Science 332, 336 (2011).

[28] M. F. Parsons, F. Huber, A. Mazurenko, C. S. Chiu, W. Setiawan, K. Wooley-Brown, S. Blatt, and M. Greiner, SiteResolved Imaging of FermionicLi6in an Optical Lattice, Phys. Rev. Lett. 114, 213002 (2015).

[29] S. Murmann, A. Bergschneider, V. M. Klinkhamer, G. Zürn, T. Lompe, and S. Jochim, Two Fermions in a Double Well: Exploring a Fundamental Building Block of the Hubbard Model, Phys. Rev. Lett. 114, 080402 (2015).

[30] C. S. Chiu, G. Ji, A. Mazurenko, D. Greif, and M. Greiner, Quantum State Engineering of a Hubbard System with Ultracold Fermions, Phys. Rev. Lett. 120, 243201 (2018).

[31] L. Tarruell and L. Sanchez-Palencia, Quantum simulation of the hubbard model with ultracold fermions in optical lattices, C. R. Phys. 19, 365 (2018).

[32] L. Pezzé and A. Smerzi, Quantum theory of phase estimation, in Atom Interferometry, Proceedings of the International School of Physics "Enrico Fermi", edited by G. M. Tino and M. A. Kasevich (IOS Press, Amsterdam, 2014), Vol. 188, p. 691.

[33] R. Kubo, The fluctuation-dissipation theorem, Rep. Prog. Phys. 29, 255 (1966).

[34] M. Gabbrielli, A. Smerzi, and L. Pezzè, Multipartite entanglement at finite temperature, Sci. Rep. 8, 31761-3 (2018).

[35] G. Mathew, S. L. L. Silva, A. Jain, A. Mohan, D. T. Adroja, V. G. Sakai, C. V. Tomy, A. Banerjee, R. Goreti, A. V. N., R. Singh, and D. Jaiswal-Nagar, Experimental realization of multipartite entanglement via quantum fisher information in a uniform antiferromagnetic quantum spin chain, Phys. Rev. Res 2, 043329 (2020).

[36] See Supplemental Material at http://link.aps.org/supplemental/ 10.1103/PhysRevResearch.3.L032051 for details on the derivation of the quench protocol and some its features as well as a discussion of the different entanglement bounds available.
[37] N. Friis, A. R. Lee, and D. E. Bruschi, Fermionic-mode entanglement in quantum information, Phys. Rev. A 87, 022338 (2013).

[38] J. Schliemann, J. I. Cirac, M. Kuś, M. Lewenstein, and D. Loss, Quantum correlations in two-fermion systems, Phys. Rev. A 64, 022303 (2001).

[39] K. Eckert, J. Schliemann, D. Bruß, and M. Lewenstein, Quantum correlations in systems of indistinguishable particles, Ann. Phys. 299, 88 (2002).

[40] T. Popoviciu, Sur les équations algébriques ayant toutes leurs racines réelles, Mathematica 9, 129 (1935).

[41] A. Bergschneider, V. M. Klinkhamer, J. H. Becher, R. Klemt, L. Palm, G. Zürn, S. Jochim, and P. M. Preiss, Experimental characterization of two-particle entanglement through position and momentum correlations, Nat. Phys. 15, 640 (2019).

[42] B. Yang, H. Sun, R. Ott, H.-Y. Wang, T. V. Zache, J. C. Halimeh, Z.-S. Yuan, P. Hauke, and J.-W. Pan, Observation of gauge invariance in a 71-site quantum simulator, Nature 587, 392 (2020).

[43] A. Mazurenko, C. S. Chiu, G. Ji, M. F. Parsons, M. KanászNagy, R. Schmidt, F. Grusdt, E. Demler, D. Greif, and M. Greiner, A cold-atom fermi-hubbard antiferromagnet, Nature (London) 545, 462 (2017).

[44] G. Salomon, J. Koepsell, J. Vijayan, T. A. Hilker, J. Nespolo, L. Pollet, I. Bloch, and C. Gross, Direct observation of incommensurate magnetism in Hubbard chains, Nature (London) 565, 56 (2018).

[45] J. Vijayan, P. Sompet, G. Salomon, J. Koepsell, S. Hirthe, A. Bohrdt, F. Grusdt, I. Bloch, and C. Gross, Time-resolved observation of spin-charge deconfinement in fermionic hubbard chains, Science 367, 186 (2020).

[46] J. Smith, A. Lee, P. Richerme, B. Neyenhuis, P. W. Hess, P. Hauke, M. Heyl, D. A. Huse, and C. Monroe, Many-body localization in a quantum simulator with programmable random disorder, Nat. Phys. 12, 907 (2016).

[47] S. Pappalardi, A. Russomanno, A. Silva, and R. Fazio, Multipartite entanglement after a quantum quench, J. Stat. Mech.: Theory Exp. (2017) 053104.

[48] M. Brenes, S. Pappalardi, J. Goold, and A. Silva, Multipartite Entanglement Structure in the Eigenstate Thermalization Hypothesis, Phys. Rev. Lett. 124, 040605 (2020).

[49] M. Mehboudi, A. Sanpera, and J. M. R. Parrondo, Fluctuationdissipation theorem for non-equilibrium quantum systems, Quantum 2, 66 (2018). 